\title{
Warming up und Cooling out in der Wissenschaft. Zur Entwicklung von Möglichkeitshorizonten am Beispiel von Wissenschaftskarrieren in Deutschland
}

\author{
Oliver Berli
}

Angenommen: 4. Oktober 2021 / Online publiziert: 18. Oktober 2021

(C) Der/die Autor(en) 2021

Zusammenfassung Mit seinem 1952 veröffentlichten Aufsatz „On cooling the mark out" formulierte Erving Goffman einen einflussreichen Vorschlag, um die Enttäuschung von Erwartungen sowie Strategien der Vermeidung und Bearbeitung typischerweise individuell zugerechneter Misserfolge zu konzeptualisieren. In kompetitiven Feldern, die zugleich Universalismus wie Leistungsgerechtigkeit versprechen und systematisch an der Einlösung dieser modernen Versprechungen scheitern, ist die Bearbeitung von enttäuschten Erwartungen eine Daueraufgabe. Ein Musterbeispiel für diese Kombination von feldspezifischem Glauben an die Relevanz von Leistung und hoher Dichte an Erwartungsenttäuschungen stellen Karrieren im Wissenschaftsfeld dar. Der Artikel nimmt am Beispiel von Wissenschaftskarrieren eine Erweiterung des Cooling-out-Konzepts um Prozesse des Warming up vor. Während Cooling out sich auf die Abkühlung von Erfolgserwartungen bezieht, zielt Warming up auf den Prozess des Aufbaus von Erfolgserwartungen. Auf der Grundlage von 20 erwerbsbiografischen Interviews mit Wissenschaftler*innen aus den Disziplinen BWL, Geschichte und Physik geht der Beitrag dem Management von Erfolgserwartungen und der Anpassung von Möglichkeitshorizonten nach und arbeitet zentrale Strategien heraus, die dabei zum Einsatz kommen.

Schlüsselwörter Wissenschaftliche Karrieren · Wissenschaftssoziologie · Erwartungen $\cdot$ Erfolg $\cdot$ Misserfolg

Oliver Berli $(\bowtie)$

Department für Erziehungs- und Sozialwissenschaften, Erziehungs- und Kultursoziologie, Universität zu Köln, Gronewaldstr. 2, 50931 Köln, Deutschland

E-Mail: oberli@uni-koeln.de 


\title{
Warming up and cooling out in science. On shifts of horizons of possibility in academic careers in Germany
}

\begin{abstract}
In his classic 1952 article "On cooling the mark out", Erving Goffman proposed an influential heuristic for analyzing the disappointment of expectations as well as possible strategies for avoiding and dealing with failure (which, typically, is attributed to individual mistakes). In competitive fields that simultaneously promise universalism and meritocracy while systematically failing to deliver on these modern promises, dealing with disappointments is an ongoing task. Academic careers are a prime example of this combination of field-specific belief in merit and a high density of setbacks. Following the example of academic careers, the article amends the cooling out concept to include processes of warming up. While cooling out refers to the cooling of ambitions, warming up aims at the process of building up ambition and career plans. On the basis of 20 qualitative interviews with researchers from the disciplines of business administration, history and physics, the article explores the strategies of managing expectations as well as the adjustment of horizons of possibility in this process.
\end{abstract}

Keywords Academic careers - Sociology of science - Expectations · Success · Failure

\section{Warming up et cooling out dans le monde scientifique. Sur l'évolution de l'horizon des possibles à partir de l'exemple des carrières scientifiques en Allemagne}

Résumé Dans son article publié en 1952 « On cooling the mark out », Erving Goffman a formulé une proposition influente pour conceptualiser la déception des attentes ainsi que les stratégies d'évitement et de gestion de l'insuccès mis sur le compte des individus. Dans les champs concurrentiels promettant à la fois l'universalisme et la méritocratie tout en échouant systématiquement à accomplir ces promesses modernes, la gestion des attentes déçues est une tâche permanente. Les carrières dans le champ scientifique constituent un exemple typique de conjonction entre la croyance dans le mérite propre à un champ et une densité élevée de déception des attentes. À partir de cet exemple, cet article entreprend de compléter le concept de cooling out par les processus de warming up. Tandis que cooling out se réfère au refroidissement d'espoirs de réussite, warming up désigne le processus de construction des espoirs de réussite. Sur la base d'entretiens portant sur la biographie professionnelle de 20 scientifiques dans les disciplines de l'économie, de l'histoire et de la physique, cet article se penche sur la gestion des espoirs de réussite et l'adaptation de l'horizon des possibles et met en évidence les principales stratégies employées dans ce contexte.

Mots-clés Carrières scientifiques · Sociologie des sciences · Attentes · Réussite · Insuccès 


\section{Einleitung}

Wissenschaftskarrieren sind hochgradig riskant. Nicht ohne Grund hat Max Weber (1988) in seiner Rede „Wissenschaft als Beruf“ diesen Karriereweg als Hasard bezeichnet. Historische Analysen belegen seine Einschätzung (Schmeiser 1994); gleichzeitig haben sich die Chancenstrukturen deutlich geändert (Kahlert 2013; Reuter et al. 2016). So hat das Missverhältnis zwischen der Zahl derjenigen, die formal für eine dauerhafte Position in der Wissenschaft qualifiziert sind, und denjenigen, die eine solche erreichen, quantitativ betrachtet in vielen Ländern zugenommen. In besonderem Maße gilt dies für Deutschland, da hier der Mittelbau ausgebaut wurde und befristete Beschäftigungsverhältnisse die Regel sind (vgl. Enders 1996; Kreckel und Zimmermann 2014). Vor diesem Hintergrund kann das Feld der Wissenschaft mit einigem Recht als Winner-takes-it-all-Markt beschrieben werden (Rogge 2015), der entgegen des feldspezifischen universalistischen Versprechens soziale Ungleichheiten perpetuiert (Engler 2001; Graf 2015; Keil 2018). Charakteristisch für das gegenwärtige wissenschaftliche Feld ist zudem, dass es in mehrfacher Hinsicht hochkompetitiv ist. Neben der Konkurrenz um Positionen, die einen dauerhaften Verbleib in der Wissenschaft ermöglichen, ist hier insbesondere an die Forschungsförderung und den Publikationsmarkt zu denken. Dass unter den genannten Bedingungen die Erfolgserwartungen von Wissenschaftler*innen regelmäßig enttäuscht werden, kann nicht ausbleiben. Eine Möglichkeit, diese Erfolgserwartungen zum Gegenstand der Analyse zu machen, besteht darin, unterschiedliche Muster der Selbsteinschätzung der Karrierechancen zu untersuchen (vgl. Funken et al. 2015; Rogge 2015). In einem alternativen Zugriff ließen sich auch Muster der beruflichen Identität und Erfolgsorientierung von Wissenschaftler*innen unterscheiden (Matthies 2016; Matthies und Torka 2019). Anstatt habituell verfestigte Muster zu untersuchen, möchte ich im Folgenden vorschlagen, den Fokus auf die Deutung und Bearbeitung von Erfolgserwartungen zu legen. Konzeptionell angeregt wurde diese Perspektive durch Erving Goffmans Aufsatz „On cooling the mark out“ (1952), der die status- und identitätsbedrohende Enttäuschung von Erwartungen sowie die Strategien der Anpassung und Bearbeitung eines typischerweise individuell zugerechneten Scheiterns zum Gegenstand hat. Goffmans Vorschlag wurde an unterschiedlichen empirischen Fällen erprobt und ausgearbeitet. Die Bandbreite reicht vom Abkühlen von Aspirationen in Bildungseinrichtungen (Clark 1960) über die Strategien von Frauen, unerwünschte Avancen im Nachtleben abzuwehren (Snow et al. 1991), bis hin zum Erwartungsmanagement bei Whale-watching-Touren (Nutch 2007). Die thematische Heterogenität dieser Beispiele kann als Hinweis auf die Elastizität der Cooling-out-Heuristik gelesen werden. Gleichwohl deutet Goffman selbst an, dass es Konstellationen gibt, in denen das Abkühlen von Erwartungen häufiger vorkommt als in anderen. Als Beispiel hierfür nennt er Ausbildungseinrichtungen (Goffman 1952, S. 456f.). Dieser Hinweis lässt sich weiterverfolgen. So ist es plausibel anzunehmen, dass in kompetitiven Feldern, die den Universalismus sowie die Geltung des meritokratischen Prinzips betonen und systematisch an diesen modernen Versprechen scheitern, Cooling out besonders relevant ist (vgl. Clark 1960). Geradezu ein Musterbeispiel für diese Kombination von feldspezifischem Glauben an die Relevanz von Leistung und hoher Dichte an Erwartungsenttäuschungen stellt das Wissenschaftsfeld dar. Vor dem 
Hintergrund einer zumeist individuellen Zurechnung von Leistung und Leistungsfähigkeit macht die strukturelle Ambivalenz von Chancenstrukturen - ,[s]ituations of opportunity are also situations of denial and failure“" (ebd., S. 569) - ein ausgefeiltes Erwartungsmanagement seitens aller beteiligten Akteure notwendig.

In konzeptioneller Hinsicht soll auf der Grundlage dieser Problemstellung nicht nur das Cooling-out-Konzept aktualisiert, sondern auch eine Erweiterung der analytischen Perspektive um Prozesse des Warming up diskutiert werden. Als solche möchte ich im Folgenden Prozesse der Öffnung des Möglichkeitshorizonts bezeichnen. Diese konzeptionelle Ergänzung erscheint geboten, da die vorliegende Literatur zu Cooling out bis auf wenige Ausnahmen (z. B. Alexander et al. 2008; Baird 1971) komplementäre oder gegenläufige Prozesse vollständig vernachlässigt hat. Die Strategien der Bearbeitung, Aus- und Umdeutung von Erfolgserwartungen werde ich dabei als Erwartungsmanagement bezeichnen.

Diese Problemstellung wird im Folgenden in drei Schritten bearbeitet. Im nachfolgenden konzeptionellen Abschnitt 2 werde ich zunächst ausgewählte Befunde zu Erfolgserwartungen in Wissenschaftskarrieren diskutieren (Abschn. 2.1). Im Anschluss an diese einführenden Bemerkungen werde ich die Cooling-out-Heuristik (Goffman 1952) vorstellen (Abschn. 2.2). Da es mir nicht um eine ausführliche ideengeschichtliche Rekonstruktion geht, steht das Anregungspotenzial des Konzepts für die Analyse von Wissenschaftskarrieren im Vordergrund. Zu diesem Zweck werde ich auf ausgewählte Studien zu Cooling out in Bildungs- und Arbeitskontexten (Clark 1960; Fürst 2016; Nadai 2007) eingehen und insbesondere die analytische Leerstelle des Aufbaus von Erfolgserwartungen aufzeigen (Abschn. 2.3 und 2.4). Im Anschluss an diese konzeptionellen Vorüberlegungen werde ich die Datengrundlage und das methodische Vorgehen der Untersuchung vorstellen (Abschn. 3), auf denen der empirische Hauptteil des Beitrags fußt (Abschn. 4). Als primäres Material dienen qualitative Interviews zu Wissenschaftskarrieren in Deutschland, wobei der Fokus auf der Postdoc-Phase liegt. ${ }^{1}$ In Auseinandersetzung mit diesem Material werde ich diverse Aspekte der Prozesse des Warming up und des Cooling out in Wissenschaftskarrieren herausarbeiten. Der Beitrag schließt mit zusammenfassenden Bemerkungen zu den empirischen Ergebnissen, ihren Limitationen sowie einem Ausblick auf mögliche Erweiterungen (Abschn. 5).

\section{Erfolgserwartungen und Cooling out in Wissenschaftskarrieren}

In modernen Gesellschaften werden individuelle Karrieren nicht über askriptive Merkmale, sondern mithilfe von Konzepten wie das der persönlichen Leistung gedeutet (Corsi 2014), die sich im besten Fall in Erfolge und Positionen übersetzen lässt. Dieser Leistungsglaube kann in Bourdieu'scher Perspektive als ein Element der feldübergreifenden doxa verstanden werden (König und Berli 2012). Die Widerlegung dieses gesellschaftlichen Leistungsglaubens gehört zum ungleichheits-

\footnotetext{
1 Der vorliegende Beitrag basiert auf Interviews, die im Rahmen des Projekts ,,Vertrauen und Wissenschaftlicher Nachwuchs“ (VWiN) durchgeführt wurden. Das Projekt wurde durch das BMBF gefördert (FKZ: 16FWN003).
} 
analytischen Markenkern der Soziologie. Und in der Tat lässt sich die Geltung des Leistungsprinzips für unterschiedliche gesellschaftliche Sphären überzeugend infrage stellen (Hartmann 2002; Neckel 2001, 2014). Dies gilt beispielsweise für das Feld der Bildung wie auch für das der Wissenschaft. In beiden Feldern dient das Leistungsprinzip als Grundlage von Bewertungen und der Legitimation für Selektionen. Im Feld der Wissenschaft kommt hinzu, dass es in vielerlei Hinsicht als Winnertakes-it-all-Markt funktioniert (Rogge 2015). Prekär ist nicht nur die Konkurrenz um Professuren und Dauerstellen, sondern auch die Auseinandersetzung um andere knappe Güter wie Fördermittel und Druckseiten in renommierten Fachzeitschriften. In Kombination führen feldspezifischer Glaube und intensive Konkurrenzbedingungen dazu, dass Enttäuschungen von Erwartungen mit zum wissenschaftlichen Alltag gehören.

\subsection{Erfolgserwartungen in der Wissenschaft}

Im wissenschaftlichen Feld lässt sich eine paradoxe Beobachtung machen. Einerseits ist das Wissen um die Relevanz sozialer Faktoren für Erfolg weit verbreitet (Engler 2001, S. 238). Andererseits ist ohne den Glauben an das Leistungsprinzip ein dauerhaftes Sich-Einlassen auf die Regeln des wissenschaftlichen Feldes schwer vorstellbar. Bei aller Distanz und Ironie in der wissenschaftlichen Selbstdarstellung sind Tätigkeiten wie Förderanträge schreiben, auf Konferenzen vortragen, Manuskripte begutachten oder publizieren nur möglich mit einem Mindestmaß an feldspezifischem Glauben. Vor dem Hintergrund dieser paradoxen Situation bietet es sich an, die feldspezifischen Vorstellungen von karriererelevanten Faktoren und typische Muster von Erfolgserwartungen genauer in den Blick nehmen. So ging Joseph C. Hermanowicz in einer Untersuchung der Frage nach, welche Eigenschaften aus der Perspektive von Physiker*innen in den USA Erfolg versprechen. Mit Abstand am häufigsten wurde von den Interviewten Beharrlichkeit (persistence) als relevante Eigenschaft genannt (Hermanowicz 2006, S. 139). Hintergrund sind die vielfältigen Ablehnungen und Rückschläge, die die Physiker*innen während ihrer Laufbahn erfahren. Die Bedeutung von Beharrlichkeit wurde vor allem in Bezug auf Begutachtungsverfahren für Publikationen und Förderanträge sowie die experimentelle und theoretische Forschungsarbeit geäußert (ebd., S. 140). Ausgehend von diesem Teilbefund lässt sich die Frage stellen, wie Wissenschaftler*innen mit Ablehnungen umgehen, wenn diese so zentral sind. Darauf bietet Hermanowicz jedoch keine Antwort. Eine alternative Perspektive nehmen Christiane Funken, Jan-Christoph Rogge und Sinje Hörlin ein. Sie arbeiten in einer komparativen Studie zu Karrierebedingungen in Wirtschaft und Wissenschaft drei Muster von Erfolgserwartungen heraus. Die interviewten Wissenschaftler*innen unterscheiden sich hinsichtlich ihrer Aufstiegsorientierungen (ja versus nein) sowie ihrer Erfolgseinschätzung (optimistisch versus pessimistisch) und werden als die Hoffnungsvollen, die Fatalist*innen und die Spielverweiger*innen typologisch auf den Begriff gebracht (Funken et al. 2015, S. 174 ff.; Rogge 2015, S. 696 ff.). Dabei zeigen die Autor*innen, dass berufliche Förderung und private Unterstützung relevante Faktoren sind, die diese Typen unterscheiden. Damit sprechen sie zwei Kontexte an, in denen es zur interaktiven Bearbeitung von Erfolgserwartungen kommen kann. Ähnlich wie in der Studie von 
Hermanowicz bleibt jedoch offen, wie sich diese vollzieht. Diese Leerstelle weist auch die Studie von Monika Jungbauer-Gans und Christiane Gross auf, die die Determinanten für erfolgreiche universitäre Karriereverläufe in den Fächern Mathematik, Rechtswissenschaften und Soziologie untersucht hat. Sie zeigt, dass die jeweiligen Einflussfaktoren für die Berufung auf eine Professur - abgesehen vom Alter zum Zeitpunkt der Habilitation - im Fachvergleich zum Teil erheblich variieren. So ist beispielsweise der positive Einfluss von Mentor*innen mit hoher Reputation zwar in der Mathematik und den Rechtswissenschaften, nicht aber in der Soziologie gegeben (Jungbauer-Gans und Gross 2013, S. 86f.). Gleichzeitig bleibt offen, wie diese Mentor*innen auf Karrieren einwirken. Zudem führt die Fokussierung auf das Erreichen einer Professur dazu, dass die vielfältigen Rückschläge und Misserfolge auf diesem Weg - ebenso wie die womöglich dauerhafte Abkühlung von Karriereambitionen aus dem Blick geraten. Deshalb möchte ich im Folgenden vorschlagen, Prozesse des Cooling out und Warming up in Wissenschaftskarrieren zu untersuchen.

\subsection{Trickbetrug und Theoriebildung: Goffmans Cooling-out-Konzept}

Erstmals ausformuliert wurde das Cooling-out-Konzept von Erving Goffman (1952) in dem Aufsatz „On cooling the mark out“, dessen Titel erklärungsbedürftig ist, weil er sich der Sondersprache von Kriminellen bedient. Am Beispiel eines sogenannten „,confidence games“ erläutert Goffman eine spezifische Form des Betrugs. In diesem wird das angehende Opfer (,mark“) von einer Person kontaktiert, die eine Beziehung zu ihm aufbaut und sein Vertrauen gewinnt. Gelingt dies, wird der Zielperson eine für sie lohnende finanzielle Option nahegebracht und sie eventuell durch einen kleinen Gewinn zusätzlich motiviert. Letztendlich wird das Unternehmen zum finanziellen Nachteil des designierten Opfers ausgehen. Ist dieses nicht bereit, den Verlust als Erfahrung hinzunehmen, sind zusätzliche Maßnahmen notwendig, damit das Opfer nicht zur Polizei geht oder versucht, Rache zu nehmen. Diese Aufgabe wird vom ,cooler“ übernommen. Der Trickbetrug und sein Verlauf bilden die Grundlage für eine facettenreiche Diskussion strukturell ähnlicher Prozesse, ${ }^{2}$ an deren Ende Status- und Identitätsbedrohungen abgewehrt oder zumindest abgemildert werden müssen. Cooling out wird aus Goffmans Perspektive dann notwendig, wenn Spannungen zwischen dem Selbstbild und den Erwartungen des Akteurs auf der einen und der Logik der Situation auf der anderen Seite bestehen. Die Arbeit am Selbst nehmen die Betroffenen (zumindest initial) nicht selbst vor, sondern sie werden durch den ,cooler“ dazu angestoßen. Für diese Aufgabe kommen unterschiedliche Personen infrage. So führt Goffman sowohl in der Hierarchie Höherstehende als auch Gleichgestellte oder Freund*innen an, die aufgrund ihrer sozialen Beziehungen zur auszukühlenden Person als geeignet angesehen werden. Weiterhin ließe sich an professionelle Gruppen wie z. B. Ärzt*innen denken (ebd.,

\footnotetext{
2 Natürlich macht nicht jede Konstellation von anstehenden Identitäts- und Statusveränderungen Prozesse des Cooling out notwendig. Dies ist beispielsweise der Fall, wenn Statusveränderungen von den Betroffenen nicht als Bedrohungen anzusehen sind - so kann der aktuelle Status zugunsten eines besser bewerteten aufgegeben werden. Dies wäre z. B. in einer klassischen Amtskarriere der Fall (Mannheim 1964). Im Kontext einer solchen geregelten Laufbahnordnung würde das Ausbleiben einer Statusveränderung ein Cooling out notwendig machen.
} 
S. 458). Unabhängig davon, wer die Aufgabe übernimmt, gibt es eine Reihe von Standardmethoden des Cooling out. Dazu gehört (1) das Angebot eines Ersatzstatus ebenso wie (2) das Bieten einer erneuten Chance, den gewünschten Status zu erhalten. Diese zweite Chance kann natürlich ausgeschlagen werden, was auf Seiten der Betroffenen die Deutung ermöglicht, dass es sich um eine eigene Entscheidung handelt. Weiterhin gibt es Konstellationen, in denen (3) die Betroffenen ihre Betroffenheit voll und offen zur Schau stellen dürfen. Schließlich führt Goffman Beispiele an, in denen (4) ein (stilles) Einverständnis zwischen Betroffenen und ,cooler“ es den Erstgenannten erlaubt, das Gesicht zu wahren. Dies ist beispielsweise der Fall, wenn einer Kündigung zuvorgekommen wird. Neben diesen Standardmethoden der Abkühlung infolge einer Statusänderung lassen sich auch präventive Strategien anführen. In Bezug auf berufliche Verläufe nennt Goffman auf Seiten der Organisation die Möglichkeit der frühzeitigen Selektion, um Individuen nicht zu einem späteren Zeitpunkt - wenn sie schon mehr investiert haben - loswerden zu müssen. Eine zweite organisationsseitige Variante stellt das genaue Gegenteil dar: Sie besteht darin, die entsprechenden Personen zu dulden bzw. mitzuschleppen (,carry“) (ebd., S. 460). Auf Seiten der potenziell Betroffenen gibt es ebenfalls präventive Strategien. Dazu zählen (1) sich nicht vollständig verpflichten (,hedging“), (2) das Committment vor sich und anderen verbergen (,,secrecy“), (3) mehrere Eisen im Feuer halten und (4) eine distanzierte oder unernste Haltung zum eigenen Engagement einnehmen. Alle diese Strategien eröffnen dem Betroffenen einen Ausweg: ,in case of failure he can act as if the self that has failed is not one that is important to him“ (ebd.).

\subsection{Cooling out im Kontext von Bildung und Erwerbsarbeit}

Der Aufsatz zu Cooling out ist nicht im gleichen Maße rezipiert worden wie andere Schriften Goffmans. Gleichwohl lässt sich eine Vielzahl von Studien anführen, die diese Heuristik nutzen, um empirische Phänomene in unterschiedlichen sozialen Feldern aufzuschließen. Zu den viel zitierten Adaptionen gehört Burton R. Clarks (1960) Analyse von Bildungseinrichtungen in den USA. Für Clark ist Cooling out unter den damaligen gesellschaftlichen Bedingungen funktional notwendig. Mit dieser Ausgangsthese ist er ganz nah bei Goffman (1952, S. 456f.). Clark zufolge wird Cooling out notwendig, da es eine systemimmanente Kluft zwischen den universalistischen Selbstbeschreibungen und Ansprüchen des Bildungssystems sowie den begrenzten Erfolgspositionen gibt. ${ }^{3}$ Über das von ihm gewählte empirische Beispiel der Junior Colleges hinausgehend, zählt Clark fünf Elemente von Cooling-out-Prozessen auf, die sich auch in anderen Arbeits- und Bildungskontexten untersuchen lassen (Clark 1960, S. 574 f.). Da ist zunächst (1) eine alternative Erfolgsmöglichkeit, die den Abzukühlenden nahegelegt wird - im Kontext von Peer-Review-Verfahren könnte dies die Einreichung eines Manuskriptes bei einem anderen Journal sein.

\footnotetext{
3 Die ungleichen Chancenstrukturen, die sich mit Blick auf höhere Bildung aufzeigen lassen, werden von Clark in diesem Aufsatz nicht diskutiert. Auch wenn ich im Folgenden Ungleichheitseffekte nicht systematisch berücksichtigen werde, ist es plausibel anzunehmen, dass Cooling out kein ,neutraler“ Prozess ist, sondern mit erhöhter Wahrscheinlichkeit soziale Ungleichheiten befördert. Insbesondere mit Fokus auf geschlechtsspezifische Ungleichheiten liegen hierzu instruktive Analysen vor (z. B. Kahlert 2011).
} 
Mit der Etablierung eines alternativen Ziels geht (2) das graduelle Loslösen vom ursprünglichen Ziel einher. Diese Neuorientierung wird unterfüttert durch (3) objektive Ablehnungen, die mit unzureichenden Leistungen legitimiert werden. Im Peer Review übernehmen Gutachten oder Schreiben von Redaktionen diese Aufgabe. Ein weiteres Element stellen (4) Akteure dar, die den Prozess tröstend begleiten. So kommunizieren Zeitschriftenredaktionen zum Teil umfänglich mit den Autor*innen von abgelehnten Manuskripten und teilen ihnen nicht bloß die negative Entscheidung mit. Schließlich verweist Clark darauf, dass (5) das Vermeiden von Standards, oder vielleicht besser das Thematisieren alternativer Bewertungsstandards, ebenfalls zum Cooling out beiträgt. Da sich Clark mit Bildungseinrichtungen beschäftigt, ist die hohe Relevanz, die er Bewertungen zuschreibt, wenig überraschend. Dass Formen der Bewertung auch in Arbeitskontexten für Prozesse des Cooling out zentral sind, zeigt Eva Nadai (2007) in ihrer ethnografischen Studie über innerbetriebliche Statusbedrohungen und -veränderungen. Der Fokus liegt dabei auf den vielfältigen kleinen Formen des Scheiterns von Beschäftigten in Unternehmen, die nicht in einen Verlust der Beschäftigung münden, wohl aber bedrohlich für den eigenen Status sind. Anregend für die Analyse von Karrieren in anderen Kontexten ist Nadais Ansatz, betriebliche Leistungsbeurteilungen als Bewährungsproben zu verstehen, die auf organisationsspezifischen kulturellen Modellen von Leistung ruhen und in ein Cooling out münden können. Übertragen auf die Untersuchung von Wissenschaft legt Nadais Studie nahe, Bewährungsproben besondere Aufmerksamkeit zu schenken. Diese sind als Evaluationen der relativen Wertigkeit von Personen zu verstehen, wie sie in der Wissenschaft beispielsweise in Berufungsverfahren vorgenommen werden. ${ }^{4}$ Eine letzte Studie, die ich hier anführen möchte, befasst sich mit angehenden Autor*innen, deren belletristische Buchmanuskripte nicht veröffentlicht werden (Fürst 2016). Wie Henrik Fürst aufzeigt, variiert deren Umgang mit diesem Ereignis erheblich. So unterscheidet er vier Varianten von Cooling out anhand der beiden Fragen, ob erstens Verantwortung für die Ablehnung des Manuskripts übernommen wird und zweitens diese als Versagen interpretiert wird. Diese Perspektive lässt sich auf die Wissenschaft anwenden, da individuell zurechenbare Leistungen und Anstrengungen als Deutungsfolie für Erfolg und Misserfolg auch hier weit verbreitet sind. Typischerweise werden diese Zurechnungen mit kausalen Deutungen verbunden, wobei neben eigenen auch fremde Handlungen sowie individuell nicht zurechenbare Umstände als Deutungsoptionen infrage kommen (Berli et al. 2019). Die Art und Weise, wie kleine und große Erfolge und Misserfolge gedeutet und zugerechnet werden, beeinflusst maßgeblich die Möglichkeitshorizonte und gibt Aufschluss darüber, inwiefern die Akteure eine Diskrepanz wahrnehmen zwischen dem, was sie für sich als erreichbar annehmen, und dem, was sie erreichen (vgl. Berli 2016).

\footnotetext{
4 Das Konzept der Bewährungsprobe verweist auf das Konzept der Prüfung bzw. des Tests, wie es in der Soziologie der Konventionen Verwendung findet (Boltanski und Thévenot 2007). Diese Perspektive erkennt einerseits an, dass es eine Pluralität von Qualitätskonventionen gibt. Andererseits sensibilisiert sie dafür, dass Prüfungen die Unsicherheit über die Qualität der getesteten Objekte, Personen oder Handlungen reduzieren sollen.
} 
Der Fokus der bislang vorgestellten Studien liegt überwiegend auf Fragen der Bearbeitung enttäuschter Erwartungen und der Prävention vor Enttäuschungen in unterschiedlichsten Konstellationen. Eine Frage, die hingegen typischerweise nicht verhandelt wird, ist die nach dem Aufbau von Erwartungen. Gerade mit Blick auf Arbeitskontexte und Karriereverläufe möchte ich dafür plädieren, Cooling out in Relation zum Aufbau von Erwartungen sowie zur Öffnung des Möglichkeitshorizontes zu diskutieren und entsprechende Prozesse als Warming up zu bezeichnen. Diese Überlegung ist nicht ohne Vorbilder. So sprechen Karl Alexander und Kolleg*innen (2008) in einer bildungssoziologischen Studie von drei Aspirationsmustern: Cooling out, Warming up und Holding steady. Während Cooling out mit der Reduktion von Bildungsaspirationen gleichgesetzt wird, sehen sie Warming up als dessen Gegenteil an. Unter Holding steady schließlich fassen sie die Fälle, bei denen sich die Bildungsaspirationen nicht verändert haben. Eine ähnliche Perspektive nimmt Alexandra König (2019) ein und unterscheidet zwischen „Trauerarbeit“ und „Erweiterungsarbeit“. Trauerarbeit versteht sie im Anschluss an Bourdieu (1979, S. 189f.) als einen Anpassungsprozess, bei dem sich die subjektiven Erwartungen den objektiven Chancenstrukturen annähern. Diese Anpassung ist nicht als individuelle Leistung zu verstehen, sondern weist eine soziale Komponente auf. Bei Bourdieu zeigt sich nun dieselbe Leerstelle wie in Goffmans Cooling-out-Konzept, nämlich die Nichtberücksichtigung der Möglichkeit der Erweiterung und Öffnung des subjektiven Möglichkeitshorizonts. König schlägt vor, diese Leerstelle durch das komplementäre Konzept der Erweiterungsarbeit zu schließen. Damit bezeichnet sie die Öffnung von subjektiven Erwartungen und objektiven Chancen in Interaktion mit anderen (König 2019, S. 353 f.).

\subsection{Zwischenfazit: Cooling out und Warming up in der Wissenschaft}

Wie Hermanowicz (2006) herausgestellt hat, nimmt Beharrlichkeit einen zentralen Platz in der moralischen Ordnung der von ihm interviewten Physiker*innen ein, weil deren wissenschaftliche Arbeit von Herausforderungen und Rückschlägen aller Art durchzogen ist. Dies dürfte in unterschiedlicher Ausprägung für Wissenschaftler*innen in den verschiedensten Disziplinen gelten. Gleichzeitig finden sich in seiner Untersuchung wie auch in anderen Studien zu Erfolgserwartungen und Karriereorientierungen in der Wissenschaft nur vereinzelte Hinweise auf den Umgang mit enttäuschten Erfolgserwartungen. Diese Leerstelle lässt sich mittels des Coolingout-Konzepts von Goffman bearbeiten. Es schärft den Blick für die Prozesse, Kontexte und Methoden der Bearbeitung von enttäuschten Erwartungen, für die dabei beteiligten Akteure und ihre Beziehungen. Mit Blick auf Erfolgserwartungen und Karrieren im hochkompetitiven Feld der Wissenschaft muss dieser Ansatz jedoch weiter präzisiert und ergänzt werden. Die Literatur zu Cooling-out-Prozessen in Arbeits- und Bildungskontexten sensibilisiert für zwei weiterführende Aspekte: Zum einen ist dies die Relevanz von Bewertungen und Bewertungsstandards (Clark 1960) sowie von Bewährungsproben (Nadai 2007). Diese treten in Wissenschaftskarrieren in vielfältiger Gestalt auf, weshalb es lohnenswert erscheint, ihre Bedeutung für die Vermeidung und Bearbeitung von Bedrohungen des wissenschaftlichen Selbst näher zu beleuchten. Zum anderen sollte im Anschluss an die Studie von Fürst (2016) der 
Frage nachgegangen werden, welche kausalen Zurechnungen die Betroffenen - aber auch andere Akteure aus ihrem Umfeld - für Erfolge und Misserfolge vornehmen und wie sich diese auf den Möglichkeitshorizont auswirken. Neben einer erhöhten Aufmerksamkeit für diese Aspekte schlage ich deshalb eine konzeptionelle Erweiterung des Blickes auf das Erwartungsmanagement von Wissenschaftler*innen vor: Neben Prozessen des Cooling out sollen auch Prozesse des Warming up, des Aufbaus von Erfolgserwartungen in die Analyse mit einbezogen werden. Beide Prozesse sollen mit Fokus auf die Veränderung von Möglichkeitshorizonten, die beteiligten Akteure und deren Strategien des Aufbaues, der Bearbeitung und der Aufrechterhaltung von Erfolgserwartungen näher analysiert werden. Da gerade bei wissenschaftlichen Karrieren die wiederholte Enttäuschung von Erfolgserwartungen wahrscheinlich ist, ist es plausibel anzunehmen, dass das Festhalten an bestimmten Zielen - z. B. die Habilitation und die Berufung auf eine Lebenszeitprofessur - von längeren Sequenzen der Bearbeitung von enttäuschten oder (partiell) erfüllten Erfolgserwartungen begleitet wird. Deshalb empfiehlt es sich, zwischen Erfolgserwartungen und Möglichkeitshorizonten analytisch zu unterscheiden. Während sich Erstere primär auf das angestrebte Ergebnis eines Prozesses (z. B. die positive Begutachtung eines Manuskripts im Peer-Review-Verfahren) beziehen, markieren Letztere den Grenzbereich zwischen dem, was als erreichbar, und dem, was als unwahrscheinlich betrachtet wird. Erfolgserwartungen in Arbeitskontexten können zudem sehr unterschiedliche Bezugsobjekte aufweisen. In der Wissenschaft kann es darum gehen, ein innovatives Thema in einem Vortrag zu lancieren, einen Laborversuch aufzubauen, ein Manuskript in einer angesehenen Zeitschrift zu platzieren oder auch innerhalb der kommenden zehn Jahre auf eine Professur zu gelangen. Diese Beispiele unterscheiden sich hinsichtlich ihres zeitlichen Horizonts, ihrer sozialen Reichweite (d. h. wie viele Akteure sind davon betroffen) und schließlich auch darin, welche Aspekte wissenschaftlicher Karrieren (z. B. die Reputation in der ,scientific community“) primär angesprochen werden. Vor dem Hintergrund eines mehr oder minder expliziten und erfahrungsbasierten Wissens über das wissenschaftliche Feld und ihre eigene Disziplin verfügen Wissenschaftler*innen zu jedem gegebenen Zeitpunkt ihrer Karrieren über einen Möglichkeitshorizont, in dessen Grenzen sie agieren, Projekte entwerfen oder Bewerbungen schreiben. Dieser Möglichkeitshorizont ist nicht auf einen erfahrungsbasierten, wissenschaftlichen Habitus zu reduzieren, da die Verfügbarkeit von Zeit und anderen Ressourcen sowie die Bedingungen vor Ort den Horizont von Wissenschaftskarrieren ebenfalls mitbedingen, beispielsweise indem sie die Entwicklung eines eigenständigen, individuellen Forschungsprogramms erschweren oder begünstigen (vgl. Laudel und Bielick 2018). Der analytische Mehrwert der hier umrissenen Perspektive soll im Folgenden anhand von empirischen Fällen aufgezeigt werden.

\section{Daten und Methoden}

Die verwendeten Daten entstammen einem Forschungsprojekt zu Wissenschaftskarrieren in Deutschland. Zu Beginn der Feldforschung wurden Gruppendiskussionen mit Wissenschaftler*innen unterschiedlicher Karrierestufen in BWL, Geschichtswissenschaft und Physik durchgeführt. Diese hatten explorativen Charakter und er- 
laubten erste Einblicke in fachspezifische Vorstellungen von Karrierewegen und -erfordernissen. In dieser ersten Phase bestätigte sich die Vorannahme, dass die drei genannten Fächer hinsichtlich ihrer Wissens- und Karrierekulturen relevante Unterschiede aufweisen. So unterscheiden sich beispielsweise Geschichtswissenschaft und BWL deutlich im Hinblick auf das durchschnittliche Alter bei der Erstberufung auf eine Professur wie auch bei der Durchlässigkeit in den nicht-wissenschaftlichen Arbeitsmarkt (BuWiN 2021, S. 190 ff.). Auf Basis dieser Phase der Feldexploration und vorliegender fachbezogener Studien (Burren 2010; Hermanowicz 1998, 2009; Knorr Cetina 1984; Weber 1984) wurden Leitfäden für erwerbsbiografische Interviews entwickelt. Im Anschluss hieran wurden deutschlandweit potenzielle Interviewpartner*innen recherchiert und per E-Mail kontaktiert. Die Recherche erfolgte in mehreren Phasen und orientierte sich an den Erfahrungen und ersten Auswertungen der bereits erfolgten Interviews. Diese Strategie basiert auf einem iterativzyklischen Verständnis des Forschungsprozesses. Sie dokumentiert sich unter anderem darin, dass sich im Projektverlauf ein starker Fokus auf Personen in der PostdocPhase ergeben hat. Diese Karrierephase ist deshalb von besonderem Interesse, da eine erste Bewährungsprobe - die Promotion - bereits absolviert wurde. Damit gehen objektive Veränderungen des Möglichkeitshorizonts einher. Zu denken ist hier u.a. an die Möglichkeit, Anträge bei der DFG und anderen Förderorganisationen zu stellen. Gleichzeitig stellen sich bestimmte Anforderungen an Wissenschaftskarrieren - wie die Entwicklung eines als eigenständig wahrnehmbaren Forschungsprofils - in dieser Phase verstärkt. Insgesamt wurden 20 erwerbsbiografische Einzelinterviews mit Wissenschaftler*innen durchgeführt. Diese wurden aufgezeichnet und anschließend für die Auswertung transkribiert. Für alle Befragten liegt zudem ein Kurzfragebogen vor, in dem standardisierte Daten (Familienstand, Personalkategorie etc.) erfasst wurden.

Für den Beitrag wurden die vorliegenden Interviews einer erneuten Analyse unterzogen. Im Fokus der Materialsichtung standen Erzählungen und Berichte über Prozesse, die Auswirkungen auf die Erfolgserwartungen sowie Möglichkeitshorizonte der Interviewten hatten. Dies waren beispielsweise Erzählungen über die Genese der Promotionsabsicht, Berichte über den Umgang mit Enttäuschungen - beispielsweise bei gescheiterten Stellenbewerbungen - oder auch Interviewpassagen, in denen es um den sogenannten Plan B ging. Die ausgewählten Fälle aus allen drei untersuchten Disziplinen wurden mit einem kodierenden Zugriff und softwaregestützt (MAXQDA) analysiert. Dabei wurde auf die Verfahrensvorschläge der Grounded Theory (Mey und Berli 2019; Strauss 1987) zurückgegriffen. Besonders dichte Passagen - dicht im Hinblick auf die Thematisierung von Karriereentwürfen, Erfolgserwartungen und deren Anpassung - wurden offen kodiert und einer detaillierten „microscopic analysis“ (Strauss 2004) unterzogen. Im Fallvergleich wurden sodann die Elemente von Cooling out und Warming up in Wissenschaftskarrieren näher bestimmt (z.B. die Strategien der Umdeutung von enttäuschten Erfolgserwartungen, die beteiligten Akteure etc.). 


\section{Warming up und Cooling out im Kontext von Wissenschaftskarrieren}

Im Folgenden werde ich das Warming up und Cooling out im Kontext von Wissenschaftskarrieren mit Bezug auf drei Schwerpunkte diskutieren. Zunächst werde ich die Erweiterung des Möglichkeitshorizonts durch Prozesse des Warming up thematisieren. Dabei werde ich zwei Varianten ansprechen: erstens Warming up im Kontext von direkter Ansprache und Förderung durch Dozierende sowie zweitens Warming up im Kontext von Bewährungsproben (wie der Promotion). Daran anschließend werde ich den Fokus auf das Erwartungsmanagement legen. Darunter fallen Strategien der Enttäuschungsprävention (z.B. Distanzierung von strategischem Karrierehandeln) ebenso wie Strategien des Umdeutens von Misserfolgen (z.B. keine individuelle Verantwortung für das Scheitern eines Projektantrags annehmen). Abschließend werde ich die Einengung des Möglichkeitshorizonts und Prozesse des Cooling out in den Blick nehmen.

\subsection{Erweiterung des Möglichkeitshorizonts durch Prozesse des Warming up}

Prozesse des Warming up können sich mit Bezug auf unterschiedliche Erfolgsziele (z.B. dauerhafte Position in der universitären Forschung) wie auch in unterschiedlichen Konstellationen vollziehen. So kann beispielsweise die direkte Ansprache und Förderung durch Lehrende sowie Betreuer*innen von Qualifikationsarbeiten zu einem Warming up beitragen. ${ }^{5}$ Im folgenden Interviewauszug berichtet eine Historikerin über ihre Bewerbung für ein Auslandsstudium:

Und dann habe ich mich aber an allen möglichen Orten beworben, also das war nur eine von vielen, und ich dachte auch nicht, dass das irgendwie realistisch wäre, so, aber ich habe das gemacht, weil eine wissenschaftliche Mitarbeiterin damals in [Universitätsstadt] zu mir gesagt hat: „,Du, da bewirbst du dich und da bewirbst dich und da bewirbst dich und da schickst auch mal eine Bewerbung zu."(Geschichte, w, promoviert)

Zeitlich bezieht sich die Interviewte auf die Phase kurz vor Abschluss ihres ersten Studiums. Der Plan für ein weiterführendes Auslandsstudium bestand schon, bevor eine wissenschaftliche Mitarbeiterin der Interviewten konkrete Ratschläge für ihre Bewerbungsstrategie gegeben hat. Dieser Entwurf wurde durch die Intervention der Mitarbeiterin nicht grundsätzlich infrage gestellt, sondern in seiner Ausführung modifiziert. Konkret erweiterte die Interviewte den Pool der Universitäten, an denen sie sich bewarb, um renommierte Standorte. Ohne die relativ klaren Hinweise, wo sie sich zu bewerben habe, hätte sie ihr Auslandsstudium mit einiger Wahrscheinlichkeit an einem Ort mit weniger Renommee absolviert. Denn im weiteren Verlauf des Interviews wird deutlich, dass ihr eine Bewerbung an der Universität, die sie schließ-

\footnotetext{
5 Ein hoher Anteil der Promovierenden in Deutschland hat während des Studiums Erfahrungen als studentische Hilfskraft oder Tutor*in gesammelt (vgl. Lenger 2008, S. 103 f.). Es ist plausibel anzunehmen, dass diese Erfahrung für die Genese wissenschaftlicher Karriereambitionen hochgradig relevant ist, insbesondere in den Fällen, in denen Studierenden eine Stelle als Hilfskraft oder Tutor*in angeboten wurde oder sie aufgefordert wurden, sich zu bewerben.
} 
lich akzeptieren wird, damals unrealistisch vorkam. Darin dokumentiert sich auch, dass sie zum damaligen Zeitpunkt bereits über die Vorstellung einer wie auch immer gearteten Hierarchie von Universitäten verfügte, von denen einige jenseits ihres subjektiven Möglichkeitshorizonts lagen. Dies ging zunächst mit einer zurückhaltenden Bewerbungsstrategie einher. Die Ermunterung durch die Mitarbeiterin, die durch den positiven Bescheid der renommierten Universität als gerechtfertigt bestätigt wird, hat zu einer graduellen Öffnung des Möglichkeitshorizonts geführt. Strukturell ähnliche Formen der Beratung und Förderung durch Lehrende und Betreuende finden sich mehrfach in den Erzählungen der interviewten Wissenschaftler*innen über das Studium und die frühe Promotionsphase. ${ }^{6}$

Wie der Interviewauszug zeigt, kann die direkte Ansprache und Förderung darin bestehen, recht konkrete Hinweise zu bereits bestehenden Plänen (z.B. Bewerbungen) oder zu gerade laufenden Arbeiten (z.B. Manuskripten) zu geben. Daneben können Ansprache und Förderung aber auch neue Optionen ins Spiel bringen und damit bisherige Handlungs- und Karriereentwürfe infrage stellen. Im folgenden Auszug berichtet eine andere Historikerin, die zum Zeitpunkt des Interviews an ihrer Habilitation arbeitet, wie sie durch einen Dozenten auf die Idee gebracht wurde, sich auf diesen Schritt überhaupt einzulassen:

\begin{abstract}
Also es gibt ja schon auch so Leute, die schon im ersten Semester wissen, dass sie mal Professor werden wollen. Da gehörte ich nicht dazu, sondern ich habe erst mal angefangen, auf Lehramt zu studieren, und bin dann eigentlich, also wenn es jetzt um Karriereentscheidungen und so was geht, eigentlich sozusagen durch einen äußeren Impuls überhaupt in die Wissenschaft gekommen, nämlich durch einen Assistenten in der Zeitgeschichte, der offensichtlich fand, dass ich Talent habe für geschichtswissenschaftliche Arbeit, und mich gefragt hat, ob ich mir nicht vorstellen kann, zu promovieren und vielleicht in die Wissenschaft zu gehen. Also da kam überhaupt der Impuls her, sonst wäre ich wahrscheinlich als Gymnasiallehrerin irgendwo. (Geschichte, w, promoviert)
\end{abstract}

Deutlich wird in diesem Auszug zunächst eine Distanzierung von anderen Studierenden, die bereits zu Beginn ihres Studiums das Ziel artikulieren, eine wissenschaftliche Erfolgsposition anzustreben. Diese Aussage ist nicht ohne innere Spannung, da die Interviewte mit der Wahl ihres Studiengangs auch ein klares Berufsziel verfolgt, nämlich das der Gymnasiallehrerin. Dieser Plan verspricht eine größere Laufbahnsicherheit als eine wissenschaftliche Karriere in der Geschichtswissenschaft. Es ist plausibel davon auszugehen, dass mit der Aufnahme eines Lehramtsstudiums ty-

\footnotetext{
${ }^{6}$ Gespräche und Diskussionen mit Personen, die eher am Spielfeldrand zu verorten sind (vgl. König 2019) - dies können beispielsweise die Eltern oder andere Akteure sein, die nicht Teil des wissenschaftlichen Feldes sind -, können bei der Abwägung von Karriereentscheidungen ebenfalls bedeutsam sein. So berichtete mir ein Postdoktorand im Interview, dass er sich bereits als Schüler für Physik interessiert habe, was aber den Erwartungen seiner Eltern entgegenlief: „Meine Eltern waren geschockt, die wollten eher, dass ich Arzt werde. Das hat sich dann erledigt, aber ja, sie hatten schon auch Sorge, dass ich dann irgendwie einen Beruf einschlage, der keine Zukunft hat." Um die Karriereambitionen der Eltern umzulenken, war u.a. ein Informationsgespräch an einer westdeutschen Universität notwendig. Dieses Beispiel macht deutlich, dass im Kontext von Karrieren auch die Erwartungen von Dritten - in diesem Fall die der Eltern mitbearbeitet werden müssen.
} 
pischerweise eine vergleichsweise starke Sicherheitsorientierung einhergeht (vgl. König 2019). Eine Promotion hingegen, zumal in der Geschichtswissenschaft, ist in dieser Hinsicht weniger eindeutig (z. B. Lenger 2008, S. $81 \mathrm{ff}$.). Im konkreten Fall geraten Promotion und eine wissenschaftliche Karriere für die Interviewte erst durch eine Intervention als Optionen in den Blick. Von einem bereits promovierten Wissenschaftler bekommt sie einen ,äußeren Impuls“. Die direkte Ansprache bietet die Möglichkeit, den bisher verfolgten Zukunftsentwurf zu hinterfragen. Die Art und Weise, wie der besagte Assistent seine Wertschätzung artikuliert hat, lässt sich aus diesem kurzen Bericht nicht erschließen. Jedoch wird deutlich, dass sowohl eine Promotion wie auch eine Wissenschaftskarriere von ihm als realistische Möglichkeiten thematisiert wurden. Dies legt die Interviewte retrospektiv als Talentförderung aus.

Dass Interventionen, wie die direkte Ansprache durch Dozierende, Einfluss auf Möglichkeitshorizonte haben können, hängt von unterschiedlichen Bedingungen ab. Die Zugehörigkeit zur Wissenschaftsgemeinschaft verbindet in den Interviews die Akteure, die am Warming up beteiligt sind. Dabei handelt es sich primär um Akteure, zu denen die Interviewten an ihren jeweiligen Universitäten Zugang hatten und mit denen sie, beispielsweise aus Lehrveranstaltungen, mehr oder weniger persönlich bekannt waren. ${ }^{7}$ Schließlich ist hier die Karrierephase zu bedenken. Diese ist deshalb von Relevanz, da sich das evaluative Repertoire von Wissenschaftler*innen im Karriereverlauf verändert. So zeigen Maximilian Fochler, Ulrike Felt und Ruth Müller (2016) am Beispiel der Lebenswissenschaften auf, dass vor dem Erlangen des Doktorgrads vielfältigere Bewertungsmaßstäbe angelegt werden als in der PostdocPhase. Übertragen auf das Warming up in Interaktion mit Dozierenden könnte dies bedeuten, dass mangels eines feldspezifischen Wissens und Möglichkeitssinns die Hinweise und Ansprache durch Dozierende ein besonderes Gewicht entfalten können. In späteren Karrierephasen hingegen, wenn sich das Gewicht unterschiedlicher evaluativer Referenzen verändert hat, ändern Interventionen durch Dritte potenziell ihre Relevanz und Funktion. Es ist plausibel anzunehmen, dass Mentor*innen oder „Stabilisatoren“ (Funken et al. 2015, S. 178) dann primär für die Aufrechterhaltung des Glaubens an die individuellen Karrierechancen benötigt werden. ${ }^{8}$

Neben der direkten Ansprache und Förderung durch Dozierende gibt es eine zweite typische Konstellation in den Erzählungen über Erweiterungen des Möglichkeitshorizonts: Bewährungsproben. Darunter lassen sich Bewertungsprozesse fassen, welche die relative Wertigkeit von Wissenschaftler*innen zum Gegenstand haben und diese mit erzeugen. In der Wissenschaft können zuvorderst Promotion und

\footnotetext{
7 Auffallend abwesend sind im empirischen Material hingegen die Partner*innen der Interviewten. In den episodischen Erzählungen zum Warming up werden sie in der Regel nicht als relevante Personen eingeführt. Ähnlich verhält es sich mit der Universitätsverwaltung, mit Coaches oder Trainer*innen. Dies könnte mit dem vorliegenden Sample zu tun haben. Zu dessen Erweiterung wäre es sinnvoll, Kontrastfälle heranzuziehen, in denen beispielsweise nach der Promotion ein Feldwechsel stattgefunden hat.

8 Gleichzeitig ließe sich die Frage stellen, inwiefern es herkunfts- oder geschlechtsspezifische Unterschiede in den Erwartungen an Mentor*innen gibt. So argumentieren beispielsweise Wofford et al. (2021, S. $9 \mathrm{ff}$.), dass Promovierende, die sich als ,first-generation students“ charakterisieren lassen, eine explizitere Anleitung von ihren Betreuer*innen erwarten, während die Promovierenden aus bildungsnahen Haushalten eher Unterstützung in der Ausbildung von Unabhängigkeit erwarten würden.
} 
Habilitation als Bewährungsproben verstanden werden. Daneben können auch Bewerbungsverfahren oder Gutachten, welche die Eignung oder Leistung einer Person evaluieren, als solche verstanden werden. Da im Rahmen dieser Verfahren Aussagen über die Wertigkeit von persönlichen Forschungsperspektiven und -kompetenzen formuliert werden, fordern diese das wissenschaftliche Selbst heraus, wenn das Ergebnis der Bewährungsprobe mit den eigenen Erwartungen kollidiert. ${ }^{9}$ Hinzu kommt bei stärker formalisierten Bewertungen wie Gutachten, Zeugnissen und Zertifikaten, dass diese als Kapital im Feld fungieren und - gestützt durch die Legitimität der jeweiligen Bewertungsverfahren - für Bewertete, Bewertende und ein wie auch immer geartetes Publikum Eignung oder auch Leistung dokumentieren. ${ }^{10}$ Das folgende Beispiel verdeutlicht die Relevanz von Bewährungsproben für die Erweiterung des Möglichkeitshorizonts. Es bezieht sich auf die positive Bewertung einer Promotion. Diese bekommt zusätzliches Gewicht durch eine zeitgleich freiwerdende Habilitationsstelle, die der Interviewte angeboten bekommt. Auf eben diese Stelle bezieht er sich eingangs:

Also, das war relativer Zufall, allerdings auch unterfüttert durch zweimal summa, dies auf die Diss gab, also einmal von meinem Chef. Das kommt wahrscheinlich häufiger vor, dass der eigene Chef das toll findet, und das andere war [Prof. X] gewesen, un das konntn wir schwerer einschätzen, wie [Prof. X] dann so benotet, und da weiß ich noch, dass ich sehr happy war, von [Prof. X] da auch ein summa zu kriegen. Also das war so ein Moment, wo ich dachte, ach, also die Wissenschaftskarriere, jetzt könnte sie so richtig starten. (BWL, $\mathrm{m}$, promoviert)

Mein Interviewpartner rahmt das Zusammenkommen von frei werdender Position an der Professur seines Erstbetreuers und zeitnahem Abschluss seiner Dissertation als zufällig. Damit wählt er eine besondere Form der kausalen Beschreibung, die weder auf sein eigenes Handeln noch auf eine Förderung durch seinen damaligen Vorgesetzten verweist. Vielmehr wird die zeitliche Koinzidenz als Zufall markiert, also einer nicht zu beeinflussenden Größe zugerechnet. Dass er für die Position infrage kommt, schreibt er aber nicht nur der zeitlichen Passung, sondern auch seiner Leistung in der Promotion zu. Die Bewertung durch seinen Erstbetreuer relativiert er mit Verweis auf dessen Rolle als Vorgesetzten, während er mit Blick auf das zweite

\footnotetext{
${ }^{9}$ Zugespitzt ließe sich formulieren, dass der Grad der Herausforderung des wissenschaftlichen Selbst davon abhängt, wie stark die Person der Wissenschaftler*in mit Gegenstand der Bewertung ist. Dies ist bei Stellenbesetzungsverfahren, persönlichen Referenzschreiben zur Teilnahme an Förderprogrammen oder Evaluationen im Rahmen von Tenure-Track-Verfahren in unterschiedlichem Maße der Fall. Jedoch können auch Gutachten im Kontext eines ,double blind“ Peer Review eine Rhetorik bemühen, die neben der zu bewertenden Arbeit auch deren Autor*in in die Waagschale wirft.

10 Allerdings muss die Verantwortung für das Ergebnis der Bewertung nicht zwangsläufig angenommen werden. Ähnlich wie Fürst (2016) in Bezug auf Schriftsteller*innen argumentiert, können Misserfolge als solche akzeptiert werden, ohne dass sie als selbst verschuldet gedeutet werden müssen. Dabei dürfte es einen Unterschied machen, ob sich die Bewertung auf ein individuell oder kollektiv zugerechnetes Erzeugnis bezieht. Wird beispielsweise ein Projektantrag negativ begutachtet, der mit mehreren Kolleg*innen erarbeitet und eingereicht wurde, lässt sich die Last der Ablehnung auf mehrere Schultern verteilen - dies kann auch in Form der wechselseitigen Zuschreibung von Verantwortung für den Misserfolg geschehen und die Enttäuschung über die Ablehnung gemeinsam bearbeiten.
} 
Gutachten das Ergebnis kaum zu antizipieren vermochte. Bis zu diesem Punkt im Interview hat mein Gegenüber mehrfach davon gesprochen, dass sein ursprünglicher Plan war, nach dem Studium der Betriebswirtschaft in die freie Wirtschaft zu wechseln. Eine wissenschaftliche Karriere war für ihn kein Thema. Dass beide Gutachter mit summa cum laude begutachteten, stößt eine Öffnung des Möglichkeitshorizonts an, welche materiell abgesichert wird durch die zeitnah frei verfügbare Habilitationsstelle. Dieses Moment der Erweiterung des Möglichkeitshorizonts, von dem mein Interviewpartner berichtet, lässt sich als Warming up deuten. In diesem Fall führt es dazu, dass ein Akteur sich auf das Risiko einer Wissenschaftskarriere einlässt, wobei das Zusammenkommen von freier Stelle und positiver Bewertung als vorläufiger Gewinn angesehen werden könnte. So stellt die angebotene Position eine komfortable Ausgangsbasis für die weitere Tätigkeit in der Wissenschaft dar, während die bestandene Promotion in Verbindung mit der sehr guten Bewertung für ihn selbst und andere seine Eignung dokumentiert.

Zusammenfassend lässt sich festhalten, dass es zwei typische Konstellationen gibt, welche eine Öffnung des Möglichkeitshorizonts in Wissenschaftskarrieren anstoßen können. Auf die Relevanz von Ansprache und Förderung durch Dozierende wurde verschiedentlich hingewiesen. So unterstreicht beispielsweise Rogge (2015, S. $699 \mathrm{ff}$.) die positiven Effekte von beruflicher Förderung für die Einschätzung der Karrierechancen. ${ }^{11}$ Die Beobachtung, dass Bewertungen und Bewährungsproben nicht nur Anlass für ein Cooling out, sondern auch für ein berufliches Warming up bieten können, verweist hingegen auf eine Leerstelle bisheriger Studien.

\subsection{Offenhalten des Möglichkeitshorizonts durch Erwartungsmanagement}

Um den Möglichkeitshorizont offenzuhalten, greifen die interviewten Wissenschaftler*innen auf unterschiedliche Strategien zurück. Ein Teil dieser Strategien kann als präventives Erwartungsmanagement verstanden werden (vgl. Goffman 1952, S. 460). Zu den im Kontext von Wissenschaftskarrieren beobachtbaren Strategien zählt, erstens, eine Kombination von zurückhaltendem und verborgenem Engagement. Diese äußert sich in einer Distanzierung vom strategischen Karrierehandeln. So fällt mit Blick auf die Selbstbeschreibungen der interviewten Wissenschaftler*innen auf, dass die wenigsten das eigene Handeln als karriereorientiert beschreiben. Diese Zurückhaltung spiegelt zum einen die Anforderungen an die Darstellung eines leidenschaftlichen wissenschaftlichen Habitus. Zum anderen erscheint eine offen artikulierte Karriereorientierung vor dem Hintergrund des deutschen Wissenschaftsfeldes und seiner Chancenstrukturen als unsichere Wette auf die Zukunft. Das entsprechende

\footnotetext{
11 In seiner historischen Studie zu deutschen Professoren unterstreicht Martin Schmeiser (1994, S. 95 ff.) die Bedeutung von sozialer Patenschaft für angehende Wissenschaftler*innen aus bildungsfernen Milieus. Auch wenn sich die Strukturen wissenschaftlicher Karrieren deutlich geändert haben, ist es naheliegend anzunehmen, dass auch gegenwärtig eine solche soziale Patenschaft besonders wichtig ist auf dem Weg zur Professur. Dies gilt m. E. insbesondere für die Öffnung von Möglichkeitshorizonten und das Festhalten an Karrierezielen über einen längeren Zeitraum hinweg.
} 
Wissen ist allen vertraut (vgl. Funken et al. 2015, S. 167). ${ }^{12}$ Neben der Strategie der Distanzierung findet sich, zweitens, das parallele Verfolgen mehrerer Erfolgsziele. Dies zeigt sich beispielsweise darin, dass parallel mehrere Stellenausschreibungen bedient werden oder aber neben der Bewerbung um eine Stelle die Einwerbung von Forschungsmitteln als Ziel verfolgt wird. Schließlich lässt sich, drittens, das eigene Commitment reflexiv in den Blick nehmen. Problematisch wird diese Strategie dann, wenn die präventiven Deutungen das Zutrauen in die Möglichkeit einer Wissenschaftskarriere unterminieren. In diesem Sinne lässt sich das folgende Plädoyer für Enttäuschungstoleranz verstehen:

Also, deshalb wollte ich nur sagen, also, um in diesem Bereich Erfolg zu haben, man muss, man kann es auch positiv sagen, man muss, ich habe jetzt gesagt, man muss ein bisschen blöd sein, man könnte aber auch positiv sagen, man muss ein dickes Fell haben. Sie müssen, sie dürfen niemals die Überzeugung verlieren, dass sie es können. Wenn sie das verlieren, wenn sie irgendwann nicht mehr davon überzeugt sind, dass sie tatsächlich jemand sind, der das, der irgendwann eines Tages da Erfolg haben wird, dann wird es, glaube ich, sehr schwer. Also, Selbstzweifel dürfen sie nicht so sehr viel zulassen. (Geschichte, $\mathrm{m}$, promoviert)

Die Strategien des präventiven Erwartungsmanagements müssen im Zusammenspiel mit potenziellen Erwartungsenttäuschungen gesehen werden. Diese Strategien erlauben es, eigene wie auch fremde Erwartungen mit Blick auf karriererelevante Ereignisse so zu rahmen, dass das wissenschaftliche Selbst nach Möglichkeit nicht in Bedrängnis gerät. Damit können sie dazu beitragen, den Möglichkeitshorizont offenund an Entwürfen und Zielen festzuhalten. Erwartungsmanagement ist jedoch nicht nur im Modus der Vorsorge beobachtbar, sondern auch als Nachsorge, d.h. wenn sich Erfolgserwartungen nicht erfüllen. Im folgenden Interviewauszug berichtet ein habilitierter Historiker von seinen bisherigen Erfahrungen mit Berufungsverfahren:

Ja viel kann ich da nicht sagen, äh, ich bewerb mich meistens [für] gar nix, manchmal Schriften einreichen, ganz selten Vorsingen, äh, und dann halt ich eben nen Vortrag und komme in der Regel nicht auf die Liste, so. (Geschichte, $\mathrm{m}$, habilitiert)

Die bisherige Bilanz fällt in der Darstellung des Interviewten ernüchternd aus. Entsprechende Erfahrungen können unterschiedlich gerahmt werden, sie tangieren aber nicht zwangsläufig den Möglichkeitshorizont, wie die lakonische Bemerkung des zitierten Historikers zeigt. Er lässt durchscheinen, dass er sich bereits einige Male auf eine Professur beworben hat. Der damit einhergehende Anspruch ist nicht ohne Grundlage. Zum einen dokumentiert seine Habilitation, dass er nach der Promotion erfolgreich ein zweites thematisches Feld erschlossen hat und damit eine der rele-

\footnotetext{
12 Gleichzeitig trifft aber auch zu, dass von den Einzelnen schon zahlreiche Investitionen in die Wissenschaftskarriere getätigt wurden, sie sowohl eine zunehmend eigenständige Forschungsagenda entwickeln als auch darauf hinarbeiten, ihren Status in der ,scientific community“ zu festigen und auszubauen. Entsprechend ist eine Wissenschaftskarriere längst Teil ihres Möglichkeitshorizonts, unabhängig davon, wie realistisch dies im Einzelnen vor dem Hintergrund der verfügbaren Stellen in den kommenden Jahren ist.
} 
vanten Anforderungen für eine Berufung in den Geschichtswissenschaften erfüllt. Diese zeichnen sich nicht nur durch eine vergleichsweise hohe Habilitationsquote aus, sondern auch durch eine weiterhin hohe Relevanz von Monografien (BuWiN 2021, S. $190 \mathrm{ff}$.). Zum anderen hat er sich in der Vergangenheit bereits erfolgreich um Drittmittel bemüht, wie im Interview deutlich wird. Seine ernüchternde Bilanz zum Zeitpunkt des Interviews übersetzt sich nicht in eine Einengung seines Möglichkeitshorizonts oder die Entwicklung alternativer Karriereziele. Vielmehr wird er sich bei der nächsten ausgeschriebenen Professur, die seinem Profil hinreichend entspricht, wieder bewerben. Dazu ist eine Deutung der bisher gescheiterten Bewerbungen notwendig, welche seine individuelle Eignung für die Wissenschaft nicht dauerhaft infrage stellt. ${ }^{13}$ Diese Strategien des Erwartungsmanagements - im Sinne eines Holding steady - sind theoretisch wie empirisch interessant, da an ihnen deutlich wird, dass auch das Festhalten an Karriereentwürfen mit Arbeit verbunden ist. Wie sieht das konkret aus? Im folgenden Auszug berichtet derselbe Interviewpartner von seinen Erfahrungen mit abgelehnten Förderanträgen:

Ähm abgelehnt worden sind, sind Druckkostenzuschüsse, äh, und Projekte, die ich mit anderen gemeinsam mache. Da hatt ich, glaub ich, zwei, da haben wir viel Zeit reingesteckt, und die sind dann abgelehnt worden, und da war es dann so, bei der Kritik, dass man sagen konnte, okay, kann man akzeptiern. Hätte man nicht ablehnen müssen, kann man aber auch ablehnen, und dann ist die Frustrationstolera-, also der Frust ist dann nicht ganz so groß. (Geschichte, m, habilitiert)

Der interviewte Historiker geht nicht näher auf die abgelehnten Druckkostenzuschüsse ein, sondern führt an, dass zwei gemeinschaftlich verfasste Förderanträge negativ entschieden wurden. Die Konstellation, mit mehreren Autor*innen einen Antrag verfasst zu haben, ist nicht unwesentlich für die Bearbeitung der Enttäuschung. Denn als Gruppe gescheitert zu sein, fordert nur bedingt das wissenschaftliche Selbstbild heraus, wenn das Scheitern individuell nicht anerkannt wird (Fürst 2016). Zudem verknüpft der Interviewte seine persönliche Reaktion auf die Ablehnung mit deren sachlicher Begründung. Mit dieser Betonung der Sachdimension wird die abschlägige Entscheidung, trotz der investierten Arbeitszeit, als legitim anerkannt. Damit dies möglich ist, müssen sich das evaluative Repertoire der Gutachtenden und der Begutachteten möglichst decken. Es ist plausibel anzunehmen, dass dies in späteren Karrierephasen wahrscheinlicher ist (Fochler et al. 2016). Neben der Karrierephase sind weitere Elemente für die erfolgreiche Umdeutung relevant. So können z. B. die Begründungen für Förderentscheidungen - neben eventuellen präventiven Rahmungen - als Bausteine für die Bearbeitung und Deutung von enttäuschten Erfolgserwartungen fungieren. Beispielsweise ermöglichen quantitative

\footnotetext{
13 Über die Anpassung des Möglichkeitshorizonts nachzudenken, eröffnet Anschlussmöglichkeiten für eine stärker ungleichheitsanalytische Perspektive (vgl. Engler 2001; Graf 2015; Keil 2018). Zum Beispiel ließe sich fragen, ob es so etwas wie herkunftsspezifische Unterschiede dessen gibt, was in den Karrierespielen als statthaft gilt. Denkbar ist, dass beispielsweise soziales Kapitel mit unterschiedlicher Selbstverständlichkeit genutzt wird, um das eigene Vorankommen zu unterstützen. So legen Studien zu ,firstgeneration students“ (Lehmann 2009) nahe, dass diese klassenspezifische moralische Dispositionen aufweisen.
} 
Angaben zur Anzahl der eingegangenen Bewerbungen oder Anträge eine Rechtfertigung des Misserfolgs mit Verweis auf die Chancenstrukturen. ${ }^{14}$ Vermittelt über eine entsprechende Deutung der enttäuschten Erfolgserwartungen lässt sich dann an den Zielen und Entwürfen festhalten oder lassen sich diese anpassen, ohne das wissenschaftliche Selbst grundlegend infrage zu stellen.

\subsection{Einengung des Möglichkeitshorizonts und Prozesse des Cooling out}

Der Möglichkeitshorizont von Wissenschaftler*innen entwickelt sich im Karriereverlauf, er kann sich sukzessive erweitern oder verengen. Relevant dafür sind vor allem die Kumulation gleichgerichteter positiver oder negativer Ereignisse (vgl. Berli et al. 2019). Neben einer zeitlichen Komponente - im Sinne einer wahrgenommenen zeitlichen Nähe - lässt sich davon ausgehen, dass starke Kontraste zwischen diesen Ereignissen und den eigenen Erwartungen zu bearbeitungswürdigen Spannungen führen können. Dies ist beispielsweise dann der Fall, wenn der Möglichkeitshorizont zunächst erweitert wurde und infolge einer anschließenden Kumulation von Erwartungsenttäuschungen die Karriereambitionen wieder reduziert, Forschungsvorhaben ad acta gelegt oder Manuskripte nicht publiziert werden. Analog zu Prozessen des Warming up sind auch im Cooling out zwei Konstellationen von besonderem Interesse. Zum einen ist dies die Beziehung zu Förder*innen und Mentor*innen, zum anderen sind es die Erfahrungen in Bewertungsprozessen und insbesondere Bewährungsproben. Im folgenden Interviewauszug berichtet mein Gegenüber lebhaft, dass er sich für sein Habilitationsprojekt relativ strategisch ein thematisches Feld gesucht hat, das gerade im Kommen und zugleich noch wenig durch Publikationen bearbeitet war:

[...] da war ich dann eine Zeitlang auch sehr rührig, was Publikationen anging, und hatte nen Auslandsaufenthalt [...], und das sollte ne Habil werden, international vergleichend [...]. Und als ich von dort zurück kam und mit Begeisterung so meine Erkenntnisse zu Papier gebracht habe, da habe ich zum ersten Mal, dass das für unsre Standardzeitschriften ziemlich uninteressant war. Also ich fand das hochspannend, ne, wie entwickelt sich so ein Konzept, also das war ja fast ne, so ne Art historische Betrachtung [...], und die Zeitschriften haben mir die Artikel quasi zurückgeschickt, also die fandn es wenig, wenig spannend. Also da gings, also zeitgleich haben dann andere Leute irgendwie so Befragungen gemacht, ne, mit 200 Unternehmen [...], und das ein bisschen multivariat ausgewertet, und das wurde abgedruckt, während diese eher historische Herangehensweise, das fanden die gar net so interessant, ähm, genau. Und da bin ich auch zum ersten Mal selbst ins Zweifeln gekommen, ob ich da glücklich

\footnotetext{
14 Neben der selektiven Transparenz kann auch die diskrete Weitergabe von Informationen für eine Umdeutung des Misserfolgs herangezogen werden. So berichten beispielsweise mehrere Interviewte über Stellenbesetzungsverfahren, in denen sie sich nicht durchsetzen konnten. Wie sich herausstellte - ob dies der Wahrheit entspricht ist unerheblich - waren die erfolgreichen Personen mit den personalverantwortlichen Professor*innen bekannt oder gar befreundet. Dadurch wird der Misserfolg zwar nicht weniger ärgerlich, aber die Verunsicherung der persönlichen Eignung wird abgewehrt, und an dessen Stelle tritt ein skandalisierbarer Modus der Stellenbesetzung.
} 
werde, in dem, in dem Umfeld, ob ich das liefern kann, was die Szene so will, gleichzeitig gabs auch son Paradigmenwechsel, dass ja die Art, wie mein Chef groß geworden ist, das würde ja heute überhaupt net mehr zählen. (BWL, m, promoviert)

In dieser retrospektiven Betrachtung werden unterschiedliche Dinge deutlich. Zunächst zeigen sich die hohen Erwartungen, die mit dem Habilitationsprojekt verbunden waren. Da ist zunächst die rege Publikationsaktivität zu nennen, wobei die Formulierung recht unbestimmt ist. Der Forschungsaufenthalt diente der Datenerhebung in zwei Ländern. Zurück in Deutschland investiert mein Interviewpartner Zeit, um das in eine Form zu gießen, die er gleichermaßen als relevant und publikationsfähig ansieht. Aus den Reaktionen der „Standardzeitschriften“ für sein Fach liest er $\mathrm{ab}$, dass sich zwischen seiner Begeisterung für das Thema, seinen Auswertungen und den Reputation versprechenden Publikationsmöglichkeiten eine deutliche Kluft auftut. Mehr noch: Den Peer-Review-Prozess überstehen Manuskripte aus Projekten, die sowohl konzeptionell als auch methodisch deutlich anders ausgerichtet sind. Diese Erfahrung verknüpft er in seinem Bericht mit Zweifeln an seiner Passung für eine wissenschaftliche Erfolgsposition. Somit beschreibt der Interviewte hier, wie er auf eine Professur hinarbeitet, ohne diesem Ziel näher zu kommen. Seine Arbeit an der Habilitation und an Manuskripten für Fachzeitschriften ist strategisch grundiert, führt aber nicht zum intendierten Erfolg. Hinzu kommt der Zweifel daran, ob das Karriereziel wirklich so erstrebenswert ist („ob ich da glücklich werde“). Mein Interviewpartner rahmt die angepasste Einschätzung seiner Karrierechancen durch den Hinweis auf geänderte Rahmenbedingungen für Karrieren in seinem Fach. Als Beleg für den „Paradigmenwechsel“ führt er an, dass die Karriere seines Vorgesetzten - den er im Verlauf des Interviews mehrmals als wichtige Identifikationsfigur benennt - unter den aktuell geltenden Bedingungen nicht wiederholbar sei. Im Rückblick formuliert er, dass er unsicher wurde, die Erwartungen der „Szene“ bedienen zu können. In der Tat haben sich die Reputationskriterien in der BWL in den vergangenen Jahrzehnten verändert (vgl. am Beispiel der Schweiz: Burren 2010, S. 198 ff.). Was hier aus der zeitlichen Distanz abgeklärt und reflektiert berichtet wird, stellt zugleich den Einstieg in einen langwierigen Anpassungsprozess dar, der in einer Einengung des Möglichkeitshorizonts mündet. Mein Interviewpartner ist immer noch in der Wissenschaft tätig, aber von dem Ziel Professur hat er nach diesen und anderen Erfahrungen - wie im Verlauf des Interviews deutlich wird - Abstand genommen. Seine Position zum Zeitpunkt unseres Gesprächs steht im Einklang mit dem Selbstbild, das er vermittelt. Dies betrifft beispielsweise seine Einschätzung der Vor- und Nachteile von Professuren, die er im Interview darlegt. Gesenkt haben sich also vor allem seine Aspirationen in Bezug auf seine Position innerhalb der Universität. In Bezug auf seine Themen ist er seinem Habilitationsprojekt treu geblieben und findet für seine Schwerpunkte auch Anerkennung, sodass er weiterhin forscht, publiziert und lehrt. Nun allerdings ohne eine Professur anzustreben.

Eingedenk der Chancenstrukturen im gegenwärtigen Wissenschaftsfeld ist eine Einengung des Möglichkeitshorizonts für viele promovierte Wissenschaftler*innen wahrscheinlich. Im obenstehenden Interviewauszug finden sowohl der damalige Vorgesetzte als auch Bewertungsprozesse Erwähnung, als wir im Interview über das 
Habilitationsprojekt und dessen Bearbeitung sprechen. Sie können beide für die Abkühlung von Karriereentwürfen relevant werden. Während eine Förderung durch andere Forscher*innen sich auch darin äußert, dass Entwürfe stabilisiert oder Enttäuschungen interaktiv umgedeutet werden (vgl. Funken et al. 2015, S. 178), stellt das Fehlen einer entsprechenden Unterstützung für die Bearbeitung von enttäuschten Erfolgserwartungen eine zusätzliche Herausforderung dar. Die Relevanz von Bewertungen für die Abkühlung von Ambitionen verdankt sich zum einen dem Umstand, dass ihnen zum Teil ein objektiver Charakter zugesprochen wird. Zum anderen können Misserfolge (beispielsweise abgelehnte Manuskripte oder Förderanträge) auch negative Auswirkungen auf die weitere wissenschaftliche Arbeit haben, indem sie - ohne einen für andere sichtbaren Ertrag - Zeit und andere Ressourcen binden bzw. gebunden haben.

\section{Zusammenfassung und Ausblick}

Vor dem Hintergrund des paradoxen Leistungsglaubens und der unsicheren Chancenstrukturen im wissenschaftlichen Feld erscheinen Mechanismen der Abkühlung von Erfolgserwartungen und Karriereambitionen als funktionale Notwendigkeit. Eine alleinige Fokussierung auf Prozesse des Cooling out erzeugt jedoch eine konzeptionelle Leerstelle: Die Seite des Aufbaus von Erfolgserwartungen wird typischerweise vernachlässigt. Die hier vorgestellte empirische Analyse stellt einen Versuch dar, diese Leerstelle zu bearbeiten. Am Beispiel von Wissenschaftskarrieren in Deutschland habe ich (1) die Erweiterung, (2) das Offenhalten und (3) die Einengung von Möglichkeitshorizonten in den Blick genommen. Bevor ich auf konzeptionelle Anschlussmöglichkeiten zu sprechen komme, möchte ich zunächst zentrale Beobachtungen aus dem empirischen Teil meines Beitrags rekapitulieren.

(1) Mit Blick auf die Erweiterung des Möglichkeitshorizonts in Prozessen des Warming up lassen sich zwei typische Konstellationen identifizieren. Zum einen sind im Kontext von Wissenschaftskarrieren primär Dozierende, Vorgesetzte sowie Betreuer*innen von Qualifikationsarbeiten an Prozessen des Warming up beteiligt. Ihre Interventionen können zur Modifikation von Handlungsentwürfen führen (z.B. Anpassung von Bewerbungsstrategien), aber auch neue Optionen (z.B. eine Promotion anstelle des zweiten Staatsexamens) denkbar werden lassen. Zum anderen können auch Erfahrungen im Kontext von Bewertungsprozessen zur Erweiterung des Möglichkeitshorizonts beitragen. Bewertungen können dabei unterschiedliche Formen annehmen (z. B. Lob und Kritik im Kolloquium, Gutachten usw.). Besonders relevant für die Öffnung (und ggf. Schließung) von Möglichkeitshorizonten sind Bewährungsproben (wie Promotion oder Habilitation). Diese zeichnen sich dadurch aus, dass Leistung individuell zugerechnet wird, auch wenn die Objekte der Bewertung (z. B. Qualifikationsschriften) das Ergebnis kollektiver Arbeit sind. Diese Prüfungssituationen objektivieren mittels der Anwendung von komplexen Verfahren und zum Teil konkurrierenden Bewertungskriterien die relative Wertigkeit von (angehenden) Wissenschaftler*innen. Hinzu kommt, dass absolvierte Bewährungsproben - beispielsweise in Stellenbesetzungsverfahren - als Kapital fungieren können. 
Auch deshalb sind sie mit Blick auf die Entwicklung von Möglichkeitshorizonten im Karriereverlauf von analytischem Interesse.

(2) Das Offenhalten von Möglichkeitshorizonten erfordert Erwartungsmanagement. Präventives Erwartungsmanagement umfasst alle Strategien, die im Falle des Eintretens eines Misserfolgs helfen, die enttäuschten Erfolgserwartungen zu bearbeiten (vgl. Goffman 1952, S. 460). Dies kann beispielsweise dadurch erreicht werden, dass die Relevanz einer Einwerbung eines Drittmittelprojekts für die weitere Forschung im Vorfeld bereits heruntergespielt wird, bevor über den Antrag entschieden wurde. Erwartungsmanagement greift aber auch dort, wo bereits Erwartungen enttäuscht wurden. Besonders plastisch wird dies, wenn - um im Beispiel zu bleiben - die Verantwortung für das Scheitern eines Förderantrags nicht als eigenes Versagen gedeutet wird. Ähnlich wie im Warming up können auch hier signifikante Andere, die der „scientific community“ angehören, von besonderer Bedeutung sein. Inwiefern „Zwischenrufe vom Spielfeldrand“ (König 2019) durch signifikante Andere, die nicht zum Feld der Wissenschaft gehören, zum Offenhalten des Möglichkeitshorizonts beitragen können, ist eine empirisch offene Frage. Mit Blick auf Bewährungsproben und andere Bewertungsformate lässt sich festhalten, dass das Offenhalten des Möglichkeitshorizonts auch davon abhängt, dass Zeit und andere Ressourcen für die Forschung bereitstehen.

(3) Im Kontext von Wissenschaftskarrieren sind - strukturell analog zum Warming up - zwei Aspekte besonders relevant für die Einengung von Möglichkeitshorizonten. Dies sind zum einen die Beziehungen zu Förder*innen und zum anderen die Erfahrungen in Bewertungsprozessen. Es ist plausibel anzunehmen, dass mit fortdauernder Zugehörigkeit zum Wissenschaftsfeld die Erstgenannten ihre Funktion ändern und nunmehr relevant für die Stabilisierung von Karriereentwürfen werden (Funken et al. 2015; Rogge 2015). Fehlen unterstützende Beziehungen, ist davon auszugehen, dass dies das Offenhalten des Möglichkeitshorizonts erschwert. Dies gilt insbesondere im Falle einer Kumulation von enttäuschten Erfolgserwartungen. Zudem ist davon auszugehen, dass Bewertungen im Karriereverlauf zunehmend relevant werden. Hierfür ist zu bedenken, dass sich die Akteure im Laufe ihrer Zugehörigkeit zum Wissenschaftsfeld immer stärker an feldinternen Qualitätskonventionen orientieren (vgl. Fochler et al. 2016). Wenn es vor diesem Hintergrund nicht gelingt, Misserfolge den ungünstigen Chancenstrukturen oder anderen Faktoren (z. B. missgünstigen Gutachtenden) zuzurechnen, stellen diese eine Herausforderung für das wissenschaftliche Selbst dar. Im Zusammenspiel mit anderen Kontextbedingungen (z. B. rechtliche Bedingungen der Weiterbeschäftigung) können solche Herausforderungen zu einer Abkühlung der Karriereambitionen beitragen.

In der Gesamtschau ermöglicht eine kombinierte Analyse von Cooling-out- und Warming-up-Prozessen ein facettenreiches Bild der Entwicklung von und der Arbeit an Möglichkeitshorizonten in Wissenschaftskarrieren, das in mehrfacher Hinsicht mit bereits vorliegenden Untersuchungen und Konzepten verknüpfbar ist. Im hier vorgeschlagenen Zugang werden Zukunftsperspektiven und Karriereambitionen von Wissenschaftler*innen nicht typologisch festgeschrieben, sondern stärker hinsichtlich ihrer Dynamik und Genese betrachtet. Die vorgeschlagene Perspektive erlaubt es zudem, auch das „Durchhalten“ bzw. Offenhalten des Möglichkeitshorizonts als Kombination unterschiedlicher Strategien des Erwartungsmanagements darstellbar 
zu machen, die nur in Interaktion mit Anderen gelingen kann. Anschlussfähig und erweiterbar ist die vorgelegte Analytik sowohl in Richtung fachkultureller als auch geschlechts- oder herkunftsspezifischer Differenzen des Erwartungsmanagements. Mit Blick auf herkunfts- und geschlechtsspezifische Effekte lassen sich Verbindungen zu habitusanalytischen Arbeiten herstellen (z. B. Engler 2001; Matthies und Torka 2019). Angesichts der oben unterstrichenen Relevanz von Bewertungen und Bewährungsproben könnten zudem fachkulturelle Unterschiede in den jeweiligen „Bewertungskulturen“ (Berli et al. 2021) mit Berücksichtigung finden (z. B. Hessels et al. 2019). Schließlich ermöglicht die hier vertretene Perspektive dem Umstand Rechnung zu tragen, dass Wissenschaftskarrieren mehrdimensional sind (Laudel und Gläser 2008). Welche Karrieredimensionen von Cooling out bzw. Warming up betroffen sind und welche Wechselwirkungen sich verzeichnen lassen, sind lohnenswerte Fragen, die hier jedoch nur andeutungsweise verfolgt werden konnten. In diese Richtung lässt sich die vorliegende Perspektive gewinnbringend weiter ausbauen und mit Studien zur Entwicklung von individuellen Forschungsprogrammen in Beziehung setzen (Laudel und Bielick 2018).

In methodischer Hinsicht sind Strategien des Erwartungsmanagements nicht ohne weiteres zugänglich. Erstens ist schwer zu entscheiden, was der Position geschuldet ist, aus der das Gegenüber spricht. Mein Hauptmaterial waren Interviews mit Angehörigen des akademischen Mittelbaus an Universitäten. Es ist eine empirisch offene Frage, ob etablierte Professor*innen im gleichen Maße präventive Deutungen mobilisieren. Unsere Gesprächspartner*innen befanden sich noch „mitten im Kampf“, wie es einer von ihnen formulierte. Zweitens ist das hier verwendete Datenmaterial auf die Perspektive der Betroffenen beschränkt. Die Strategien der ,cooler“ und Mentor*innen lassen sich „über Bande“ - d.h. über episodische Erzählungen und Berichte in den Interviews - nur begrenzt erfassen. Vor dem Hintergrund dieser Überlegungen würde sich eine Ergänzung durch Interviews mit erfahrenen Professor*innen - erfahren als ,gatekeeper“, Mentor*innen und Betreuer*innen - anbieten. Dabei sollten sie als ,cooler" wie auch als Förderer*innen in den Blick genommen werden (z. B. Beaufaÿs 2004, S. 233 ff.; Funken et al. 2015, S. 176 ff.). Gleichermaßen könnte eine Beschäftigung mit schriftlichen Bewertungskommunikationen als Element des Cooling out und Warming up lohnenswert sein. Eine Option wäre, die Kommunikation zwischen Fachzeitschriften bzw. Förderorganisationen und Autor*innen bzw. Antragstellenden zu untersuchen. Eine solche Erweiterung der Untersuchungsperspektive hätte den Vorteil, an bestehende Vorarbeiten zum Peer Review anknüpfen zu können (z. B. Reinhart 2012), und könnte zugleich neues relevantes Material für die Herausforderung und Behauptung wissenschaftlicher Selbstbilder zutage fördern.

Danksagung Mein Dank gilt den anonymen Gutachter*innen für ihre kritischen Nachfragen und hilfreichen Anregungen sowie allen, die frühere Versionen meines Textes kommentiert haben. Besonders gedankt sei an dieser Stelle Hildegard Matthies, Heike Kahlert sowie Alexandra König, die mir mehrfach mit konstruktiven Kommentaren zur Seite standen.

Funding Open Access funding enabled and organized by Projekt DEAL.

Open Access Dieser Artikel wird unter der Creative Commons Namensnennung 4.0 International Lizenz veröffentlicht, welche die Nutzung, Vervielfältigung, Bearbeitung, Verbreitung und Wiedergabe in 
jeglichem Medium und Format erlaubt, sofern Sie den/die ursprünglichen Autor(en) und die Quelle ordnungsgemäß nennen, einen Link zur Creative Commons Lizenz beifügen und angeben, ob Änderungen vorgenommen wurden.

Die in diesem Artikel enthaltenen Bilder und sonstiges Drittmaterial unterliegen ebenfalls der genannten Creative Commons Lizenz, sofern sich aus der Abbildungslegende nichts anderes ergibt. Sofern das betreffende Material nicht unter der genannten Creative Commons Lizenz steht und die betreffende Handlung nicht nach gesetzlichen Vorschriften erlaubt ist, ist für die oben aufgeführten Weiterverwendungen des Materials die Einwilligung des jeweiligen Rechteinhabers einzuholen.

Weitere Details zur Lizenz entnehmen Sie bitte der Lizenzinformation auf http://creativecommons.org/ licenses/by/4.0/deed.de.

\section{Literatur}

Alexander, K., Bozick, R., \& Entwisle, D. (2008). Warming up, cooling out, or holding steady? Persistence and change in educational expectations after high school. Sociology of Education, 81, 371-396.

Baird, L. L. (1971). Cooling out and warming up in the junior college. Measurement and Evaluation in Guidance, 4(3), 160-171.

Beaufaÿs, S. (2004). Wie werden Wissenschaftler gemacht? Beobachtungen zur wechselseitigen Konstitution von Geschlecht und Wissenschaft. Bielefeld: transcript.

Berli, O. (2016). Der Wandel subjektiver Möglichkeitshorizonte und die Genese von Karriereambitionen. Zur empirischen Problematisierung exemplarischer Theorien wissenschaftlicher Karrieren. In J. Reuter, O. Berli \& M. Tischler (Hrsg.), Wissenschaftliche Karriere als Hasard. Eine Sondierung (S. 337-360). Frankfurt a. M.: Campus.

Berli, O., Hammann, B., \& Reuter, J. (2019). Wissenschaftskarriere als Glückspiel? Zur Karriererelevanz von Glück aus professoraler Sicht. Beiträge zur Hochschulforschung, 41(4), 114-134.

Berli, O., Nicolae, S., \& Schäfer, H. (2021). Bewertungskulturen. Ein Vorschlag für eine vergleichende Soziologie der Bewertung. In O. Berli, S. Nicolae \& H. Schäfer (Hrsg.), Bewertungskulturen (S. 1-21). Wiesbaden: Springer VS.

Boltanski, L., \& Thévenot, L. (2007). Über die Rechtfertigung. Eine Soziologie der kritischen Urteilskraft. Hamburg: Hamburger Edition.

Bourdieu, P. (1979). Die feinen Unterschiede. Kritik der gesellschaftlichen Urteilskraft. Frankfurt a. M.: Suhrkamp.

Burren, S. (2010). Die Wissenskultur der Betriebswirtschaftslehre. Aufstieg und Dilemma einer hybriden Disziplin. Bielefeld: transcript.

BuWiN (2021). Bundesbericht Wissenschaftlicher Nachwuchs 2021. Statistische Daten und Forschungsbefunde zu Promovierenden und Promovierten in Deutschland. Bielefeld: wbv.

Clark, B. R. (1960). The „,ooling-out“ function in higher education. American Journal of Sociology, 65, $569-576$.

Corsi, G. (2014). Hypertrophie der Zukunft. Scheitern als Perspektive der Karriere. In J. René \& A. Langhof (Hrsg.), Scheitern - Ein Desiderat der Moderne? (S. 301-321). Wiesbaden: Springer VS.

Enders, J. (1996). Die wissenschaftlichen Mitarbeiter. Ausbildung, Beschäftigung und Karriere der Nachwuchswissenschaftler und Mittelbauangehörigen an den Universitäten. Frankfurt a. M.: Campus.

Engler, S. (2001). „In Einsamkeit und Freiheit?“ Zur Konstruktion der wissenschaftlichen Persönlichkeit auf dem Weg zur Professur. Konstanz: UVK.

Fochler, M., Felt, U., \& Müller, R. (2016). Unsustainable growth, hyper-competition, and worth in life science research: Narrowing evaluative repertoires in doctoral and postdoctoral scientists' work and lives. Minerva, 54, 175-200.

Funken, C., Rogge, J.-C., \& Hörlin, S. (2015). Vertrackte Karrieren. Zum Wandel der Arbeitswelten in Wirtschaft und Wissenschaft. Frankfurt a. M.: Campus.

Fürst, H. (2016). Handling rejection as failure: Aspiring writers getting the rejection slip. Valuation Studies, $4,153-176$.

Goffman, E. (1952). On cooling the mark out: Some aspects of adaptation to failure. Psychiatry, 15, $451-463$.

Graf, A. (2015). Die Wissenschaftselite Deutschlands. Sozialprofil und Werdegänge zwischen 1945 und 2013. Frankfurt a. M.: Campus. 
Hartmann, M. (2002). Der Mythos von den Leistungseliten. Spitzenkarrieren und soziale Herkunft in Wirtschaft, Politik, Justiz und Wissenschaft. Frankfurt a. M.: Campus.

Hermanowicz, J. C. (1998). The stars are not enough: Scientists - Their passions and professions. Chicago: University of Chicago Press.

Hermanowicz, J. C. (2006). What does it take to be successful? Science, Technology, \& Human Values, 31, 135-152.

Hermanowicz, J. C. (2009). Lives in science: How institutions affect academic careers. Chicago: University of Chicago Press.

Hessels, L. K., Franssen, T., Scholten, W., \& De Rijcke, S. (2019). Variation in valuation: How research groups accumulate credibility in four epistemic cultures. Minerva, 57, 127-149.

Jungbauer-Gans, M., \& Gross, C. (2013). Determinants of success in university careers: Findings from the German academic labor market. Zeitschrift für Soziologie, 42, 74-92.

Kahlert, H. (2011). „Cooling out“ und der riskante Weg an die Spitze - Zum Einfluss von Ungleichheitsregimes auf Karriereorientierungen im wissenschaftlichen Nachwuchs. In J. Wergen (Hrsg.), Forschung und Förderung. Promovierende im Blick der Hochschulen (S. 105-123). Münster: Lit.

Kahlert, H. (2013). Riskante Karrieren. Wissenschaftlicher Nachwuchs im Spiegel der Forschung. Opladen: Verlag Barbara Budrich.

Keil, M. (2018). Zur Reproduktion sozialer Ungleichheit im Feld der Wissenschaft. Berliner Journal für Soziologie, 28, 457-478.

Knorr-Cetina, K. (1984). Die Fabrikation von Erkenntnis. Zur Anthropologie der Naturwissenschaft. Frankfurt a. M.: Suhrkamp.

König, A. (2019). Spielfelder des Selbst. Eine Längsschnittstudie zu jungen Erwachsenen in Handwerksbetrieben, Hochschulen und Kunstakademien. Weinheim: Beltz Juventa.

König, A., \& Berli, O. (2012). Das Paradox der Doxa - Macht und Herrschaft als Leitmotiv der Soziologie Pierre Bourdieus. In P. Imbusch (Hrsg.), Macht und Herrschaft. Sozialwissenschaftliche Konzeptionen und Theorien (S. 303-333). Wiesbaden: Springer VS.

Kreckel, R., \& Zimmermann, K. (2014). Hasard oder Laufbahn. Akademische Karrierestrukturen im internationalen Vergleich. Leipzig: Akademische Verlagsanstalt.

Laudel, G., \& Bielick, J. (2018). The emergence of individual research programs in the early career phase of academics. Science, Technology, \& Human Values, 43, 972-1010.

Laudel, G., \& Gläser, J. (2008). From apprentice to colleague. The metamorphosis of early career researchers. Higher Education, 55, 387-406.

Lehmann, W. (2009). Becoming middle class: How working-class university students draw and transgress moral class boundaries. Sociology, 43, 631-647.

Lenger, A. (2008). Die Promotion. Ein Reproduktionsmechanismus sozialer Ungleichheit. Konstanz: UVK.

Mannheim, K. (1964) [1930]. Über das Wesen und die Bedeutung des wirtschaftlichen Erfolgsstrebens: ein Beitrag zur Wirtschaftssoziologie. In K. Mannheim, Wissenssoziologie (S. 625-687). Berlin: Luchterhand.

Matthies, H. (2016). Akademischer Hazard und berufliche Identitäten. In J. Reuter, O. Berli \& M. Tischler (Hrsg.), Wissenschaftliche Karriere als Hasard. Eine Sondierung (S. 29-48). Frankfurt a. M.: Campus.

Matthies, H., \& Torka, M. (2019). Academic habitus and institutional change: Comparing two generations of German scholars. Minerva, 57, 345-371.

Mey, G., \& Berli, O. (2019). Grounded Theory in der Kultursoziologie. In S. Moebius, F. Nungesser \& K. Scherke (Hrsg.), Handbuch Kultursoziologie. Band 2: Theorien-Methoden-Felder (S. 243-259). Wiesbaden: Springer VS.

Nadai, E. (2007). Cooling out. Zur Aussortierung von „Leistungsschwachen“. In C. Arni (Hrsg.), Der Eigensinn des Materials: Erkundungen sozialer Wirklichkeit (S. 445-461). Frankfurt a. M.: Stroemfeld.

Neckel, S. (2001). „Leistung“ und „Erfolg“. Die symbolische Ordnung der Marktgesellschaft. In E. Barlösius, H.-P. Müller \& S. Sigmund (Hrsg.), Gesellschaftsbilder im Umbruch. Soziologische Perspektiven in Deutschland (S. 245-265). Opladen: Leske + Budrich.

Neckel, S. (2014). Die Pflicht zum Erfolg. Genealogie einer Handlungsorientierung. In D. Hänzi, H. Matthies \& D. Simon (Hrsg.), Erfolg. Konstellationen und Paradoxien einer gesellschaftlichen Leitorientierung. Leviathan, Sonderband 29 (S. 27-44). Baden-Baden: Nomos.

Nutch, F. (2007). On cooling the tourist out. Notes on the management of spoiled expectations. Qualitative Sociology Review, 3(2), 64-71.

Reinhart, M. (2012). Soziologie und Epistemologie des Peer Review. Baden-Baden: Nomos.

Reuter, J., Berli, O., \& Tischler, M. (Hrsg.). (2016). Wissenschaftliche Karriere als Hasard. Eine Sondierung. Frankfurt a. M.: Campus. 
Rogge, J.-C. (2015). The winner takes it all? Die Zukunftsperspektiven des wissenschaftlichen Mittelbaus auf dem akademischen Quasi-Markt. Kölner Zeitschrift für Soziologie und Sozialpsychologie, 65, 685-707.

Schmeiser, M. (1994). Akademischer Hasard. Das Berufsschicksal des Professors und das Schicksal der deutschen Universität 1870-1920. Stuttgart: Klett-Cotta.

Snow, D. A., Robinson, C., \& McCall, P. L. (1991). „Cooling out“ men in singles bars and nightclubs: Observations on the interpersonal survival strategies of women in public places. Journal of Contemporary Ethnography, 19, 423-449.

Strauss, A. L. (1987). Qualitative analysis for social scientists. Cambridge: Cambridge University Press.

Strauss, A. L. (2004). Analysis through microscopic examination. Sozialer Sinn, 5, 169-176.

Weber, W. (1984). Priester der Clio. Historisch-sozialwissenschaftliche Studien zur Herkunft und Karriere deutscher Historiker und zur Geschichte der Geschichtswissenschaft 1800-1970. Frankfurt a. M.: Lang.

Weber, M. (1988) [1919]. Wissenschaft als Beruf. In M. Weber, Gesammelte Aufsätze zur Wissenschaftslehre (S. 582-613). Tübingen: Mohr.

Wofford, A. M., Griffin, K. A., \& Roksa, J. (2021). Unequal expectations: First-generation and continuinggeneration students' anticipated relationships with doctoral advisors in STEM. Higher Education. https://doi.org/10.1007/s10734-021-00713-8.

Oliver Berli, Dr. phil., Wissenschaftlicher Mitarbeiter an der Professur für Erziehungs- und Kultursoziologie der Universität zu Köln und Mitbegründer des AKs „Soziologie des (Be)Wertens“ der Sektion Wissenssoziologie der DGS. Forschungsschwerpunkte: Wissens- und Wissenschaftssoziologie, Soziologie des Wertens und Bewertens, Kultursoziologie, Methodologie und Methoden der qualitativen Sozialforschung. Ausgewählte Veröffentlichungen: Grenzenlos guter Geschmack. Die feinen Unterschiede des Musikhörens, 2014; (mit J. Reuter \& M. Tischler, Hrsg.) Wissenschaftliche Karriere als Hasard. Eine Sondierung, 2016; Diszipliniertes Interpretieren. Zur Praxeologie des gemeinsamen Interpretierens qualitativer Daten, in: Soziale Welt, 2017. 